\title{
One-Stage Correction with Intra- and Extraatrial Rerouting of Anomalous Systemic and Pulmonary Venous Return and Intraventricular Repair of Double Outlet Right Ventricle in a Patient with Heterotaxy Syndrome
}

\author{
Helena Staehler ${ }^{1}$, Carina Hopfner ${ }^{2}$, Masamichi Ono ${ }^{1, *}$ and Jürgen Hörer ${ }^{1}$ \\ ${ }^{1}$ Department of Congenital and Pediatric Heart Surgery, German Heart Center Munich, Technische Universität München, Division \\ of Congenital and Pediatric Heart Surgery, University Hospital of Munich, Ludwig-Maximilians-Universität, Munich, Germany \\ ${ }^{2}$ Department of Pediatric Cardiology and Pediatric Intensive Care, University Hospital of Munich, Ludwig-Maximilians-Universität, \\ Munich, Germany \\ *Corresponding Author: Masamichi Ono. Email: ono@dhm.mhn.de
}

Received: 15 July 2021 Accepted: 16 August 2021

\begin{abstract}
We report a very rare case of successful intracardiac correction in a patient with heterotaxy syndrome. The cardiac malformations included dextrocardia, double outlet right ventricle, pulmonary stenosis, interrupted inferior vena cava, hemiazygos continuation and total anomalous pulmonary venous return. One-stage correction was performed. The atrial procedure consisted of intra- and extraatrial rerouting of the anomalous systemic and pulmonary venous return. The hepatic veins were detached and diverted to the left atrium via an extracardiac conduit. The correction of the double outlet right ventricle was accomplished by intraventricular redirection of the blood flow from the left ventricle to the aorta. The right ventricular outflow was ultimately remodeled using a valved conduit. For better perception of the complex morphology, a three-dimensional model was designed, using CT scan images. This proved to be very useful for surgical planning, especially with regard to the intraatrial reconstruction of the systemic and pulmonary venous rerouting.
\end{abstract}

\section{KEYWORDS}

Heterotaxy syndrome; anomalous systemic venous return; anomalous pulmonary venous return; azygos continuation; double outlet right ventricle; 3D modeling

\section{Introduction}

The heterotaxy syndrome can be defined as anomalous arrangement and lateralization of thoracoabdominal organs [1,2]. It is distinguished by neither displaying a situs solitus, nor presenting as a clearly mirrored image, as can be found in the situs inversus. Moreover, it comprises a great variety of visceral and cardiac malformations in most cases [3]. Therefore, all the more importance is attached to the meticulous planning of the highly individual surgical approach of each defect. Interdisciplinary conferences and broad diagnostics are indispensable. Modern techniques such as 3D modelling may help to establish a profound understanding of morphological features [4]. 


\section{Case Report}

A seven-month-old, male infant presented with situs anomaly and heterotaxy syndrome. He had been diagnosed with double outlet right ventricle (DORV) and malposition of the great arteries. Further echocardiographic and computer tomographic examination showed dextrocardia with a morphological right ventricle (RV) on the right side and morphological left ventricle (LV) on the left side. The atriums displayed an isomerism of the left appendages. Moreover, a malalignment ventricular septal defect (VSD) and left superior vena cava (SVC) with hemiazygos continuation could be detected. The patient was admitted to our clinic for an elective procedure. At admission, the patient showed signs of cardiac decompensation and increased sweating while drinking. Because of the complex intracardiac anatomy, the operative method had been discussed at our interdisciplinary conference. For this purpose, a 3D-model of the patient's heart had been created (Fig. 1).

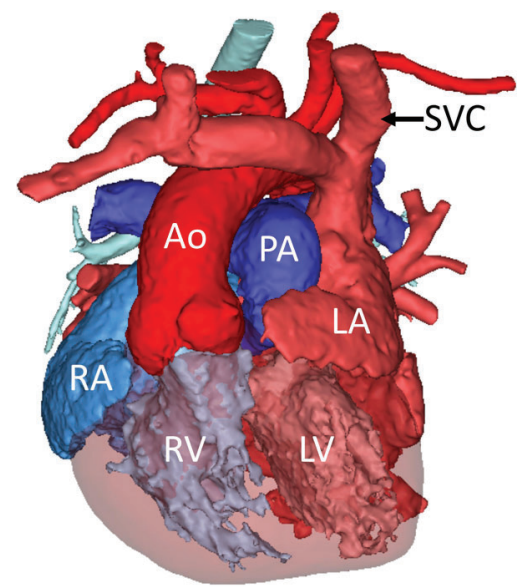

Figure 1: Preoperative 3D rendering of the patient's heart created by segmentation of a CT data set. $\mathrm{Ao}=$ Aorta, $\mathrm{PA}=$ pulmonary artery, $\mathrm{LA}=$ left atrium, $\mathrm{RA}=$ right atrium, $\mathrm{LV}=$ left ventricle, $\mathrm{RV}=$ right ventricle, SVC = superior vena cava. The myocardium is pictured transparently to allow viewing of the intracardiac structures

3D models are constructed using the Mimics Innovation Suite Medical 23.0 software (Materialise NV, Leuven, Belgium). With its help the contrast-enhanced blood pool is segmented, i.e., marked, in a CT or MRI scan of the patient. A comprehensive toolbox allows to trim the segmented area, to fill regions of poorly distributed contrast agent and to separate wrongly connected structures caused by image resolution and artifacts. A virtual 3D model representing the intracardiac surface is then generated based on the segmentation. A hollow model of the heart is designed by adding a wall of uniform thickness around the blood volume and including the myocardium. Creating openings and windows allows to view the inner structures, e.g., the septum, of the heart. To mimic the surgical procedure patches as well as conduits are digitally simulated according to the planned operation. Finally, the digitally reconstructed heart modelsblood pool (Fig. 2a) and hollow model with simulated patches and conduits (Fig. 2b) - are 3D printed using the appropriate printing technique and material.

Abdominal scanning revealed that the liver was located in the midline, while the hepatic veins returned to the left atrium (LA). The LA also received the pulmonary veins and the SVC with hemiazygos continuation. All systemic and pulmonary veins were connected to the LA. The only right atrial inlet was an atrial septal defect (ASD) of $12 \mathrm{~mm}$. The aorta arose predominantely from the right ventricle, lateral of the pulmonary artery (PA), the great vessels were arranged side by side. The aortic valve $(12-13 \mathrm{~mm})$ showed no signs of insufficiency or stenosis. The PA presented with severe subvalvular stenosis and 
blood flow acceleration of $4.5 \mathrm{~m} / \mathrm{s}$. The pulmonary valve $(6-7 \mathrm{~mm})$ was presumably bicuspid. LV and RV were connected via an inlet-outlet VSD, the outlet tract being rather small at 6-7 $\mathrm{mm}$.
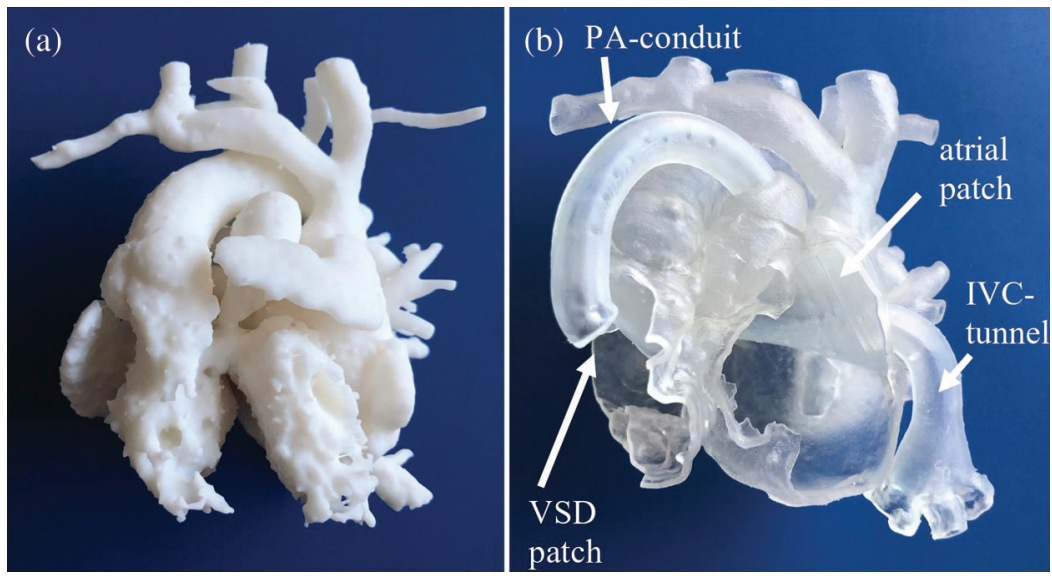

Figure 2: (a) 3D printed model representing the intracardiac surface of the patient. (printer: Form3, Formlabs $\mathrm{GmbH}$; material: White (stiff, white), Formlabs $\mathrm{GmbH}$ ), (b) 3D printed hollow model of the heart including the myocardium (printer: Agilista 3200W, Keyence Coop.; material: AR-G1L (flexible, translucent), Keyence Coop.) and 3D printed elements of preoperatively simulated surgical approach, i.e., atrial patch, VSD patch, PA-conduit, IVC-tunnel (printer: Form3, Formlabs GmbH; material: Clear (stiff, transparent), Formlabs $\mathrm{GmbH}$ )

The elective operation was performed with median sternotomy. The previously collected findings were confirmed intraoperatively. The procedure was performed with cardiopulmonary bypass and cardioplegic cardiac arrest with mild hypothermia $\left(32^{\circ} \mathrm{C}\right)$. The LA was opened, aorta and pulmonary artery were cut close to the heart. We then performed a ventriculotomy in order to inspect the VSD, which measured $10 \times 15 \mathrm{~mm}$. The distance between VSD and aortic valve was $15 \mathrm{~mm}$. The VSD was tunneled with a Goretex-patch, commencing a Rastelli-procedure. Initially we had planned to reroute the hepatic veins inside the right atrium. However, after inspecting the LA containing the hepatic veins, pulmonary veins, and the mitral valve, we decided that there would not be enough space for intraatrial tunneling. Therefore, we resected the hepatic veins from the LA and rerouted them via conduit (Goretex, $16 \mathrm{~mm}$ ) to the inferior edge of the LA-incision (Fig. 3).

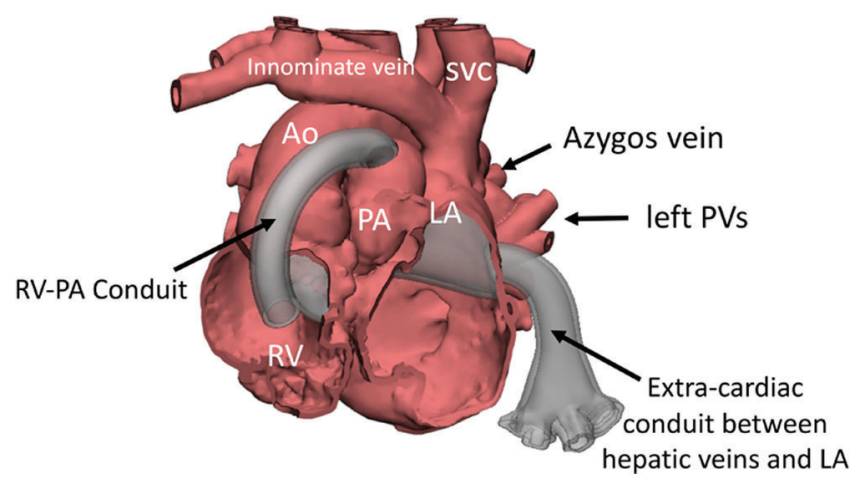

Figure 3: 3D design used to draft surgical approach. Rerouting of the hepatic veins to the right atrium was performed using an extra cardiac-conduit. Right ventricle was connected to the PA via conduit 
All systemic veins, including the hepatic venous conduit and the SVC with the hemiazygos venous flow now led to the upper part of the LA. The LA was divided into a pulmonary and a systemic venous compartment using a Goretex patch. It was attached above the pulmonary veins and below the systemic veins and the ASD (Fig. 4).

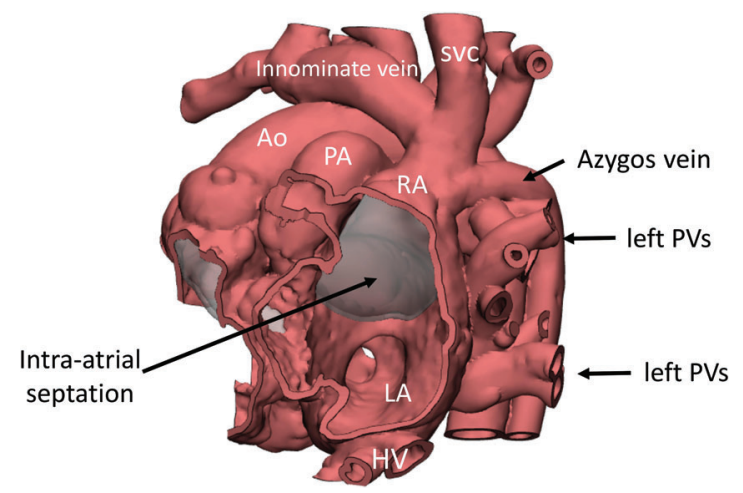

Figure 4: 3D model, designed for depiction of the intracardiac rerouting of systemic and pulmonary veins. Intraatrial view shows the Goretex patch that was used for division of the atrium

The systemic venous compartment was enlarged by closing the LA with a bovine pericardial patch. A $16 \mathrm{~mm}$ Hancock conduit was anastomosed with the central pulmonary artery and proximally attached to the right ventriculotomy. Lastly, the divided aorta was re-anastomosed. Postoperatively no AV regurgitation was detected, the biventricular function was normal. Blood flow towards the conduit was laminar with slight turbulence with the conduit without insufficiency $(\operatorname{Vmax} 1.5 \mathrm{~m} / \mathrm{s}$ ). The patient was extubated within a few hours postoperatively. Further postoperative recovery of the patient was uneventful. The patient was discharged from the ICU after nine days. We arranged a transfer to an external clinic after an inpatient stay of two weeks. Prior to discharge, echocardiography showed good biventricular function, no AV regurgitation and laminar blood flow through the Rastelli-tunnel. The RV-PA conduit was well functioning with minimal valve insufficiency. The patient was discharged with stable vital parameters.

\section{Discussion}

The uniqueness of the presented case lays in the fact that both pulmonary and systemic veins were returned to the left-sided atrium, and the blood flow to the right-sided atrium was supplied only through the atrial septal defect. Therefore, septation of the systemic and pulmonary venous blood flow was the main issue of the intracardiac correction. Several operative methods of rerouting the systemic veins to the right sided atrium were discussed beforehand. One option would have been to connect the hepatic veins to the azygos veins. However, the distance between the hepatic and the azygos veins was too large to be able to connect them. We further considered attaching the innominate vein to the right-side atrium. However, due to the large blood flow from the SVC and from the hemiazygos vein, that would have passed through the innominate vein, it was not appraised as a suitable solution. Finally, we decided to perform intraatrial rerouting of the SVC and hemiazygos flow to the right-side atrium. The hepatic veins were initially planned to be intraatrially attached to the right side of the pulmonary veins. However, intraoperatively it became apparent that there was not enough space to create a route inside the atrium. This is why we decided to perform an extracardiac diversion of the hepatic veins into the right atrium using a Goretex tube.

Additionally, both atria presented with isomerism of the left appendages. Studies demonstrated that left isomerism is most commonly associated with polysplenia [5]. Poly- and asplenia in patients with herterotaxy 
syndrome are correlated with a higher 1-year mortality [6,7]. However, our patient did not display any anomalies concerning the spleen.

3D-models play an important role in our approach. In recent years, 3D modelling has emerged as an excellent method for appraoching technically challenging cases in various surgical specialities [8]. In particular, the surgical treatment of congenital heart diseases benefits from the visualization of the individual morphology of the patient. Especially in the treatment of clinical conditions such as DORV, heterotaxy, anomalous systemic and pulmonary venous connections this technique is used [4]. The 3D reconstruction-virtual or in print-facilitates the visualization of the anatomy and thus offers new perspectives on the respective problem [9]. This also improves surgical planning, both in the conference and for the surgeon themself.

In preparation for surgery, measurements of the distance between the aortic valve and the VSD and the size of the VSD were taken in this case. The distance between the aortic valve and the VSD measured in the model was consistent with the intraoperative measurement of $15 \mathrm{~mm}$. The size of the VSD, however, was smaller in the model than in the interopertive measuring $(10 \times 12.6 \mathrm{~mm} v \mathrm{~s} .10 \times 15 \mathrm{~mm})$. This deviation may occur due to the fact that the imaging is performed on the beating heart. This can lead to a change in the position of the septum and therefore also in the size of the VSD. However, the minor difference in the measurement was negligible and did not cause any complications during the procedure. Furthermore, the current technique is limited by the fact that no rehearsal of the operation can be performed preoperatively. An improvement for the future would therefore be to use a material for printing which is compatible for cutting and suturing [10].

Further improvements to the technique could also be introduced in the creation of the conduits. Future applications of the simulation could be hemodynamic analysis by computational fluid dynamics (CFD) to determine the best shape and size for the conduit not only based on the surgeon's experience, but by utilizing additional mathematical results of the fluid flow simulation.

\section{Conclusion}

In the presented case we performed a successful intra- and extracardiac anatomical repair in a patient with heterotaxy syndrome and complex cardiac malformations. The preoperative 3D reconstruction of the patient's heart provided a considerable advantage in understanding the precise cardiac anatomy. The use of models has already found its way into our daily clinical routine.

Ethics Statement: Informed consent was obtained from legal guardians.

Funding Statement: The authors received no specific funding for this study.

Conflicts of Interest: The authors Helena Staehler, Carina Hopfner, Masamichi Ono and Jürgen Hörer declare that they have no conflicts of interest to report regarding the present study.

\section{References}

1. Agarwal, R., Varghese, R., Jesudian, V., Moses, J. (2021). The heterotaxy syndrome: Associated congenital heart defects and management. Indian Journal of Thoracic and Cardiovascular Surgery, 37(Suppl. 1), 67-81. DOI 10.1007/s12055-020-00935-y.

2. Berg, C., Geipel, A., Kamil, D., Krapp, M., Breuer, J. et al. (2006). The syndrome of right isomerism — prenatal diagnosis and outcome. Ultraschall in der Medizin, 273(3), 225-233. DOI 10.1055/s-2005-858639.

3. Jacobs, J. P., Anderson, R. H., Weinberg, P. M., Walters, H. L. III, Tchervenkov, C. I. et al. (2007). The nomenclature, definition and classification of cardiac structures in the setting of heterotaxy. Cardiology in the Young, 17(Suppl. 2), 1-28. DOI 10.1017/S1047951107001138. 
4. Yoo, S. J., Hussein, N., Peel, B., Coles, J., van Arsdell, G. S. et al. (2021). 3D Modeling and printing in congenital heart surgery: Entering the stage of maturation. Frontiers in Pediatrics, 9, 621-672. DOI 10.3389/fped.2021.621672.

5. Kim, S. J. (2011). Heterotaxy syndrome. Korean Circulation Journal, 415(5), 227-232. DOI 10.4070/kcj.2011.41.5.227.

6. Bartz, P. J., Driscoll, D. J., Dearani, J. A., Puga, F. J., Danielson, G. K. et al. (2006). Early and late results of the modified fontan operation for heterotaxy syndrome 30 years of experience in 142 patients. Journal of the American College of Cardiology, 48(11), 2301-2305. DOI 10.1016/j.jacc.2006.07.053.

7. Loomba, R. S., Nijhawan, K., Anderson, R. (2016). Impact of era, type of isomerism, and ventricular morphology on survival in heterotaxy: Implications for therapeutic management. World Journal for Pediatric and Congenital Heart Surgery, 71(1), 54-62. DOI 10.1177/2150135115601831.

8. Batteux, C., Haidar, M. A., Bonnet, D. (2019). 3D-printed models for surgical planning in complex congenital heart diseases: A systematic review. Frontiers in Pediatrics, 7, 23. DOI 10.3389/fped.2019.00023.

9. Goo, H. W., Park, S. J., Yoo, S. J. (2020). Advanced medical use of three-dimensional imaging in congenital heart disease: Augmented reality, mixed reality, virtual reality, and three-dimensional printing. Korean Journal of Radiology, 212(2), 133-145. DOI 10.3348/kjr.2019.0625.

10. Hussein, N., Kasdi, R., Coles, J. G., Yoo, S. J. (2020). Use of 3-dimensionally printed heart models in the planning and simulation of surgery in patients with Raghib syndrome (coronary sinus defect with left superior vena cava). JTCVS Techniques, 2, 135-138. DOI 10.1016/j.xjtc.2020.01.023. 